\title{
Asset Price Dynamics with Value-at-Risk Constrained Traders*
}

\author{
Jón Daníelsson Hyun Song Shin \\ Jean-Pierre Zigrand \\ Financial Markets Group \\ London School of Economics
}

June 2001

\begin{abstract}
Risk management systems in current use treat the statistical relations governing asset returns as being exogenous, and attempt to estimate risk only by reference to historical data. These systems fail to take into account the feedback effect in which trading decisions impinge on prices. We investigate the consequences for asset price dynamics of the widespread adoption of such techniques. We illustrate through simulations of a general equilibrium model that, as compared to the case when such techniques are not used, prices are lower, have time paths with deeper and longer troughs, as well as a greater degree of estimated volatility. The magnitudes can sometimes be considerable. Far from promoting stability, widespread adoption of such techniques may have the perverse effect of exacerbating financial instability.
\end{abstract}

\footnotetext{
*First version. Subsequent updates of this paper will be posted on the authors' homepages or www.RiskResearch.org. All authors are at the Financial Markets Group, and Department of Accounting and Finance of the London School of Economics. Their email addresses are, respectively, j.danielsson@lse.ac.uk, h.s.shin@lse.ac.uk and j.p.zigrand@lse.ac.uk.
} 


\section{Introduction}

The adoption of risk-management techniques by fund managers and other market participants has proceeded at a rapid pace in recent years. Their adoption has been driven not only by the market participants themselves in reaction to their experiences of market turbulence, but also by a regulatory climate that encourages putting so-called market sensitive risk management systems at the operational center of financial institutions. In particular, the 1996 amendment of the Basel Accord on regulatory capital for market risk (Basel Committee on Banking Supervision, 1996) has been especially influential in this development.

All risk-management systems in widespread use today rest on techniques that attempt to infer the statistical relations governing asset returns by reference to actual, historical realizations of returns. Indeed, this is dictated by the Basel Accord. By their nature, such risk-management systems treat the uncertainty governing asset returns as being exogenous. What they fail to take into account is the fact that the behaviour of market participants is affected by the adoption of these techniques, creating a feedback effect on the whole financial system. In short, these systems fail to take into account the system-wide consequences of widespread adoption of these techniques. Basak and Shapiro (2001), Daníelsson (2000), Daníelsson and Zigrand (2001), Morris and Shin (1999), Crockett (2000), and Persaud (2000) are some recent comments that express unease about the current practice.

The main concern is aptly summarized in the following passage ${ }^{1}$.

"So-called value-at-risk models (VAR) blend science and art. They estimate how much a portfolio could lose in a single bad day. If that amount gets too large, the VAR model signals that the bank should sell. The trouble is that lots of banks have similar investments and similar VAR models. In periods when markets everywhere decline, the models can tell everybody to sell the same things at the same time, making market conditions much worse. In effect, they can, and often do, create a vicious feedback loop."

The issues raised by this debate are eerily reminiscent of the debate in the 1970s arising from the Lucas Critique, (see Lucas, 1976). The main lesson drawn by macroeconomic forecasters then was that one should distinguish between reduced form regularities (which are liable to break down when behaviour changes) from genuinely structural relations of the economy. The

\footnotetext{
${ }^{1}$ The Economist Magazine, Oct 12th 2000.
} 
failure to distinguish properly between the two lead to unjustifiable conclusions concerning the consequences of policy. This point is succinctly summarized in Goodhart's Law:

"Any statistical relationship will break down when used for policy purposes" (Goodhart, 1974)

The blindspot in conventional risk management techniques, and especially the supervisory approach to the regulation of risk, is the presumption that risk management is a single-person decision problem - in the jargon, a game against nature. That is, uncertainty governing price movements is assumed to be exogenous, and assumed not to depend on the actions of other decision makers. The analogy is with a meteorologist trying to predict the weather. The weather is unaffected by the predictions issued by weather forecasters and the consequent actions that these forecasts generate. So, if the forecast is fine today, this will influence the actions of those decision makers for whom this information is useful, but their actions will not change the weather. Financial markets are different. When short run price changes are influenced by the trading decisions of market participants (as surely they must), then shifts in the beliefs of market participants will lead to actions that precipitate certain outcomes. There is, in other words, a feedback effect from the beliefs of market participants to the actual outcome in the market. Indeed, there are strong reasons to believe that this feedback effect will reinforce any exogenous pressures on prices arising from the fundamentals. When traders believe that prices are likely to fall, short term trading motives will induce them to sell, thereby exacerbating the price fall. Furthermore, the feedback effects have been amplified by technical advances, i.e. increased modelling of risk may worsen these adverse effects.

A gambling analogy is instructive in driving home this point. If the underlying uncertainty facing a trader were exogenous, modelling risk is akin to a gambler facing a spin of a roulette wheel, where the bets placed by him and other gamblers do not affect the outcome of the spin. However, when the outcome depends on the actions of other traders, risk modelling resembles poker more than roulette.

Current risk management practices rest on the roulette view of uncertainty. The roulette wheel may have an unknown number of outcomes with differing probabilities, but as long as the outcome is unaffected by the actions of other gamblers, it is simply a matter of applying standard statistical techniques to past outcomes to enumerate what these outcomes are, and to estimate their respective probabilities. Much of the sophisticated techniques in the current 
state of the art can be seen as alternative ways of refining such estimation procedures, as well as tracking the non-linear payoff structures arising from derivative securities. To the extent that the stochastic process governing asset prices depends on what traders do, this view of the world is invalid. The uncertainty facing traders is endogenous, and depends on the actions of market participants.

To canny traders, this is a rather obvious and banal point, and the fixed income and FX pages of the press and news services abound with references to strategic trading terms such as overhangs of leveraged positions, shortcovering and the like. So, it is clear that traders recognize the endogenous nature of market risk, even if their risk management systems do not. The neglect of the endogenous nature of risk in risk management models is all the more puzzling when set against the lessons drawn after the October 1987 crash of the stock market. The Brady 1988 Commission attributed the magnitude and swiftness of the price decline to practices such as portfolio insurance and dynamic hedging techniques. Such trading techniques have the property that they dictate selling an asset when its price falls and buying it when the price rises. Best estimates at the time suggested that around $\$ 100$ billion in funds were following formal portfolio insurance programs, representing around $3 \%$ of the pre-crash market value. However, this is almost certainly an underestimate of total selling pressure arising from informal hedging techniques such as stop-loss orders (see the survey evidence presented in Shiller, 1987). Portfolio insurance has been discussed e.g. by Grossman and Vila (1989), Gennotte and Leland (1990), Basak and Shapiro (1995), and Grossman and Zhou (1996). The events of the summer and autumn of 1998 in which financial markets experienced severe disruptions to liquidity and risk appetite can only be satisfactorily understood if the endogeneity of financial risk is taken into account.

In what follows, we investigate the consequences of the widespread adoption of market-sensitive risk management systems that treat financial market uncertainty as exogenous, and do not take into account the feedback from traders' actions to the market outcomes. We gauge their systemic impact by comparing the dynamics of asset prices by risk-management systems and compare this with asset prices dynamics in the absence of such systems. The plan of the paper is as follows. In the next section, we will give a nontechnical overview of our model and summarize some of our main simulation results. Section 3 presents the model in more detail, and Section 4 describes our simulations in more detail. 


\section{Overview of Model and Simulation Results}

Our model is based on a standard asset pricing model where a large number of small traders each have constant absolute risk aversion, but where the degree of risk aversion varies across traders. The underlying uncertainty is driven by the cashflow process which is assumed to be conditionally normal. The model builds on the single period model by Daníelsson and Zigrand (2001) in which a Value-at-Risk (VaR) constraint is introduced that restricts the portfolio choices of traders. Specifically, traders forecast next period's risk, and this estimate restricts their portfolio selection. A similar model has been proposed by Basak and Shapiro (2001). Crucially, traders follow current practices in risk management and forecast risk by means of a standard variance-covariance (VCV) technique. In other words, traders use a backward-looking belief revision process in which forecasts are generated by reference to past realizations of returns, as recommended by many of the current risk management systems and required by regulators.

When the VaR constraint binds, we show that it has an effect similar to an increase in the underlying risk aversion of the traders. Since the degree to which this constraint binds is determined by market outcomes, the net effect of the VaR constraint is that the traders behave as if their degree of risk aversion is fluctuating with the market outcomes. In particular, since the VaR constraint binds mostly during periods of market turbulence, the increased effective risk aversion leads to sales of risky assets, and serves to exacerbate market volatility and to reduce liquidity. This is indeed one way in which we can understand the frequently heard statement from market pundits during distressed episodes that the "level of risk aversion has gone up". Such statements are normally frowned upon by conventional economists, since risk aversion is part of the make-up of the individual trader (much like the colour of his eyes), and are part of the basic parameters of the economy. However, our interpretation suggests that even if the underlying basic preferences remain constant over time, the actions of the traders are determined by a rule which is identical to the actions of someone whose risk aversion is fluctuating over time.

The dynamics of the model are generated by the sequence of events in which the risk-constrained traders have a set of beliefs based upon which they form their net asset demands. Market-clearing then generates equilibrium prices. The assets yield exogenous random payoffs which in turn determine realized returns for risky assets. Completing the cycle, the traders use this realization to update their beliefs and the economy advances by one period. The following diagram illustrates the sequence of events that generate the 
dynamics in our model.

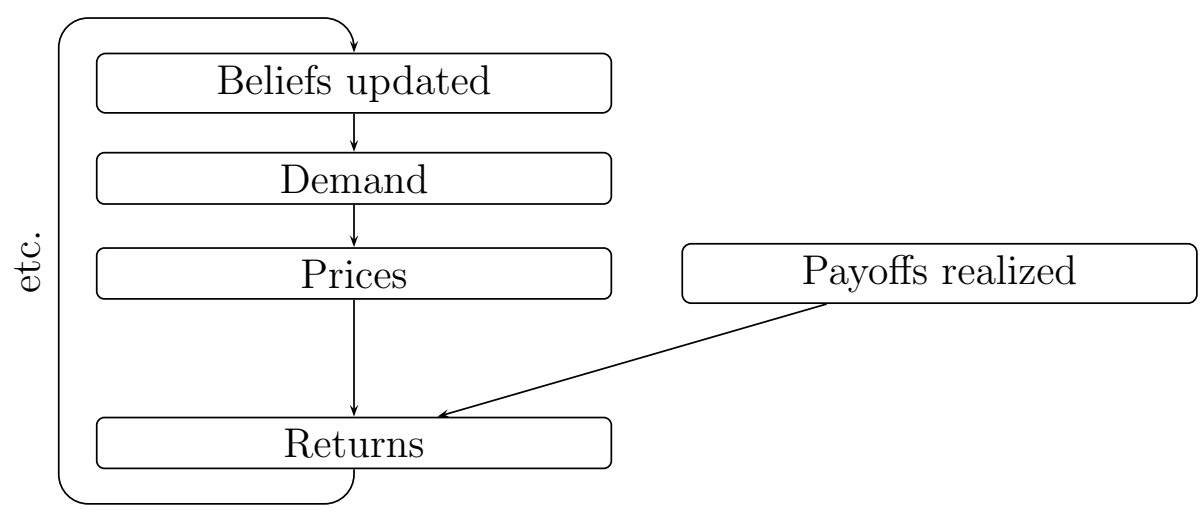

We can implement our model once we specify the exogenous stochastic process that generates the payoffs of the assets at the beginning of each date. We use standard linear normal models to generate this exogenous uncertainty, incorporating volatility clustering $(\mathrm{GARCH})$ effects, calibrated to stylized facts about financial data. Our simulations reveal the following effects of the widespread adoption of VaR constraints.

- Prices (for positive-beta assets) are lower with VaR constraints than without. The magnitudes can sometimes be very substantial.

- Following a negative shock to asset prices, the troughs in the price paths are deeper, last longer, and take a longer time to recover when there are VaR constraints. Again, the magnitudes can be very large.

- The forecast variance of returns is, in general, larger with VaR constraints than without. The difference in the forecast variance as between the constrained and unconstrained economy is largest during distressed episodes in the market.

From our simulations, it seems that the widespread adoption of backwardlooking risk management practices, such as standard VCV methods, may have a detrimental effect on asset price volatility and market distress. Far from having a dampening effect on price volatility, it may have the perverse effect of exacerbating it. These results are rather unsettling, and offer plenty of food for thought for market participants, policy makers, and academics alike. 


\section{Model}

We now give a more complete description of our model, and describe how a VaR constraint affects traders' actions. The model is a multiperiod extension of the Daníelsson and Zigrand (2001) model. Time is discrete, and indexed by $t \in\{0,1,2, \cdots\}$. In each period, there are three traded assets, labelled as assets 0,1 and 2. Asset 0 is the risk-free asset, while assets 1 and 2 are risky. Without loss of generality we assume there is a fixed, deterministic time-invariant supply of $\theta_{i}$ units of the $i^{\text {th }}$ risky asset. We denote by $q_{i t}$ the price of the $i^{\text {th }}$ asset at time $t$. Any asset traded at time $t-1$ matures at date $t$, and yields payoff $d_{i t}$. The gross return on asset $i$ between periods $t$ and $t+1$ is denoted by $R_{i, t+1}$, and defined as

$$
R_{i, t+1} \equiv \frac{d_{i, t+1}}{q_{i t}}
$$

Denote by $\boldsymbol{R}_{t}$ the vector of returns over risky assets, so that

$$
\boldsymbol{R}_{t} \equiv\left(\begin{array}{c}
R_{1 t} \\
R_{2 t}
\end{array}\right)
$$

The traders in our model have short horizons, in the sense that they choose their portfolio to maximize expected utility in the next period. The short horizon assumption tries to capture the incentives that fund managers face in which considerable emphasis is placed on short-term assessments of their performance, or internal controls on their discretion such as 'daily earnings at risk' (DEAR) limits.

To further facilitate the analysis, we reduce the dynamic portfolio choice problem to a sequence of one-period choice problems by assuming that the assets in the economy mature after one period, and traders must roll over their holding in each period. If we denote by $x_{t}^{h}$ the number of units of the safe asset held by trader $h$ between periods $t$ and $t+1$ and by $y_{i t}^{h}$ the number of units of the risky asset $i$ held by trader $h$ between periods $t$ and $t+1$, then the wealth in period $t+1$ for trader $h$ is given by

$$
W_{t+1}^{h} \equiv x_{t}^{h} d_{0, t+1}+\sum_{i} y_{i t}^{h} d_{i, t+1}
$$

At date $t$, trader $h$ aims to maximize the expected value of his von Neumann Morgenstern utility

$$
u\left(W_{t+1}^{h}\right)
$$


The traders are also assumed to have constant absolute risk aversion, so that the asset demands are independent of wealth, and prices depend only on the aggregate endowments, rather than the distribution of endowments across traders. In particular, we suppose that there is a continuum of small traders and denote by $\alpha^{h}$ the coefficient of absolute risk aversion of trader $h$. The population of traders is such that $\alpha^{h}$ is distributed uniformly on the interval $[\ell, 1]$ where $\ell>0$. This last assumption ensures that there are no risk-neutral traders in our model.

Since prices are independent of the distribution of wealth across traders in such a model, we can simplify the notation without affecting the equilibrium prices by assuming that the new supply of the risky assets at each period is owned by individuals other than the traders themselves. Moreover, we suppose that the aggregate endowments of the risky assets are constant over time. Denote by

$$
\boldsymbol{\theta} \equiv\left(\begin{array}{c}
\theta_{1} \\
\theta_{2}
\end{array}\right)
$$

the vector of time-invariant aggregate supplies of the risky assets.

\subsection{Belief Revision Process}

A key feature of our approach is the modelling of the beliefs of the traders. In order to replicate the effect of risk-management systems that rely on realized historical returns, we investigate the effects of a backward-looking belief revision mechanism for traders. We assume that the traders update their beliefs through standard VCV tools for forecasting covariance matrices, in particular RiskMetrics ${ }^{\mathrm{TM}}$. Furthermore we assume the traders use a similar forecasting rule for returns. Although it would be straightforward to model updating of beliefs with more sophisticated techniques, such as having the traders optimize a GARCH-type model each period, we believe that this would not add to our results in any significant way.

The traders' beliefs on returns are conditionally jointly normal, and we denote by $\left(\boldsymbol{\mu}_{t}, \boldsymbol{\Sigma}_{t}\right)$ the traders' beliefs concerning the expected returns $\boldsymbol{\mu}_{t}$ and covariances of returns $\boldsymbol{\Sigma}_{t}$ of the risky assets between dates $t$ and $t+1$. Thus,

$$
\begin{aligned}
& \boldsymbol{\mu}_{t}=\left(\begin{array}{l}
\mu_{1 t} \\
\mu_{2 t}
\end{array}\right) \\
& \boldsymbol{\Sigma}_{t}=\left(\begin{array}{ll}
\sigma_{t}^{11} & \sigma_{t}^{12} \\
\sigma_{t}^{12} & \sigma_{t}^{22}
\end{array}\right)
\end{aligned}
$$


where $\sigma_{t}^{i j}$ is the covariance of $R_{i, t+1}$ and $R_{j, t+1}$.

The traders adjust their beliefs concerning the expected returns and covariances by taking a geometric weighted average of past realizations, where the decay factor $\rho=0.97$ follows the recommendations of the RiskMetrics Group (1999). The backward-looking belief revision mechanism can be formalized in terms of the recursive updating rule given by the mapping

$$
\left(\left(\boldsymbol{\mu}_{t}, \boldsymbol{\Sigma}_{t}\right), \boldsymbol{R}_{t+1}\right) \mapsto\left(\boldsymbol{\mu}_{t+1}, \boldsymbol{\Sigma}_{t+1}\right)
$$

where

$$
\sigma_{t+1}^{i j}=\rho \sigma_{t}^{i j}+(1-\rho)\left(R_{i t}-\mu_{i t}\right)\left(R_{j t}-\mu_{j t}\right)
$$

for constant $\rho \in(0,1)$, and likewise for $\boldsymbol{\mu}_{t+1}$, so that

$$
\boldsymbol{\mu}_{t+1}=\rho \boldsymbol{\mu}_{t}+(1-\rho) \boldsymbol{R}_{t}
$$

The dynamics of our model are generated in the following fashion. The economy begins with an initial set of beliefs for the traders, given by $\left(\boldsymbol{\mu}_{0}, \boldsymbol{\Sigma}_{0}\right)$. Based on these beliefs, traders make their portfolio choices. If traders are constrained by a VaR constraint, their portfolio choices will be affected (we will examine the optimal portfolio rules in more detail in the following section). Given the portfolio choices, the aggregate demand functions can be defined. Together with the aggregate endowments $\boldsymbol{\theta}$, we can derive the prices of the assets. Then, the realizations of payoffs $\left\{d_{1}\right\}$ determine the returns $R_{1}$ for the risky assets, and the traders update their beliefs according to (1) and (2). This is repeated until the simulation ends at $t=T$.

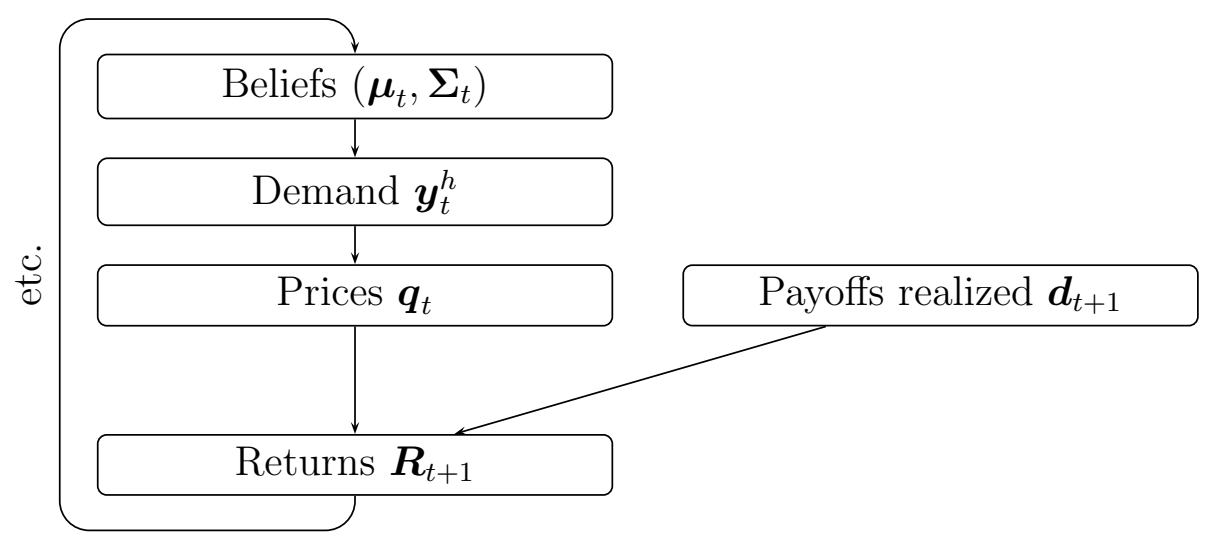

In order to implement our model, we need to solve for the optimal portfolio decisions of the traders, and specify the stochastic process generating the payoffs. We tackle each in turn. 


\subsection{The Value--at-Risk Constraint}

We first solve the portfolio choice problem of the traders, in which they face a possible constraint on the probability of a loss beyond some fixed size. We examine the constraint of the form

$$
\operatorname{Prob}_{t}\left[\mathrm{E}_{t}\left(W_{t+1}^{h}\right)-W_{t+1}^{h} \geq \mathrm{VaR}\right] \leq \bar{p}
$$

where VaR is the permitted value-at-risk, and $\bar{p}$ is the permitted probability with which the VaR limit can be breached. Given the conditional normality of beliefs, we can express this constraint in simple terms. Denoting by $\Phi(\cdot)$ the distribution function of the standard normal, note that (3) holds if and only if

$$
\Phi\left(\frac{-\mathrm{VaR}}{\sigma_{W, t+1}}\right) \leq \bar{p}
$$

where $\sigma_{W, t+1}$ is the standard deviation of $W_{t+1}^{h}$ with respect to the beliefs at date $t$. Equivalently, this condition can be written as

$$
\sigma_{W, t+1}^{2} \leq\left(\frac{\mathrm{VaR}}{\Phi^{-1}(1-\bar{p})}\right)^{2} \equiv \bar{v}
$$

Thus, the VaR constraint boils down to a constraint on the maximal permitted variance of $W_{t+1}^{h}$. The right hand side of (4) is a constant determined by the parameters of the problem, and we will denote it by $\bar{v}$.

It it useful to specially considering the real and forecast distribution of payoffs in addition to returns. The forecast distribution follows from the updating process (1). Denote by

$$
\left(\boldsymbol{m}_{t}, \boldsymbol{S}_{t}\right)
$$

the forecast mean and covariance of payoffs, and denote by

$$
s_{t}^{i j}
$$

the $(i, j)$ th entry of $\boldsymbol{S}_{t}$. Thus, $s_{t}^{i j}$ is the traders' belief of the covariance between $d_{i, t+1}$ and $d_{j, t+1}$.

We can then incorporate the VaR constraint formally into a trader's portfolio choice problem. Denoting by $\mathrm{E}_{t}(\cdot)$ the expectations operator with respect to beliefs at date $t$, trader $h$ 's optimization problem at date $t$ is to maximize

$$
\mathrm{E}_{t}\left(u^{h}\left(W_{t+1}^{h}\right)\right)
$$


by choosing portfolio holdings $x_{t}^{h}, y_{1 t}^{h}, y_{2 t}^{h}$, subject to

$$
\begin{aligned}
q_{0 t} x_{t}+\sum_{i} q_{i t} y_{i t}^{h} & \leq d_{0 t} x_{t-1}+\sum_{i} d_{i t} y_{i, t-1}^{h} \\
\sum_{i} \sum_{j} s_{t}^{i j} y_{i t}^{h} y_{j t}^{h} & \leq \bar{v}
\end{aligned}
$$

The first constraint is the period $t$ budget constraint of trader $h$. The right hand side of the constraint is the available wealth resulting from last period's investment, while the left hand side is the value of the portfolio purchased at time $t$. The second constraint is the VaR constraint, which can be expressed more succinctly as

$$
\boldsymbol{y}_{t}^{h^{\prime}} \boldsymbol{S}_{t} \boldsymbol{y}_{t}^{h} \leq \bar{v}
$$

The first-order condition for the optimal portfolio can then be written as:

$$
\mathrm{E}_{t}\left[u^{h^{\prime}}\left(W_{t+1}^{h}\right)\left(\boldsymbol{d}_{t+1}-d_{0, t+1} \boldsymbol{q}_{t}\right)\right]=2 \lambda_{t}^{h} \boldsymbol{S}_{t} \boldsymbol{y}_{t}^{h}
$$

where $\lambda_{t}^{h}$ is the Lagrange multiplier associated with the VaR constraint for trader $h$ at date $t$. Denote by $\phi_{t}^{h}$ the normalized Lagrange multiplier for the VaR constraint, given by.

$$
\phi_{t}^{h} \equiv \frac{2 \lambda_{t}^{h}}{\mathrm{E}_{t}\left[u^{h^{\prime}}\right]}
$$

Then, the first order condition can be re-arranged to yield the demand functions:

$$
\boldsymbol{y}_{t}^{h}=\frac{1}{\alpha^{h}+\phi_{t}^{h}} \boldsymbol{S}_{t}^{-1}\left(\boldsymbol{m}_{t}-d_{0, t+1} \boldsymbol{q}_{t}\right)
$$

Were it not for the $\phi_{t}^{h}$ term in the denominator, these demand functions are the familier textbook ones for traders with constant absolute risk aversion. The VaR constraint enters into the problem by raising the effective risk aversion for the trader from $\alpha^{h}$ to $\alpha^{h}+\phi_{t}^{h}$. In other words, the introduction of the VaR constraint acts in the same way as a fluctuating risk aversion level of the trader.

By setting aggregate demand equal to the supplies $\boldsymbol{\theta}$, we can solve for the equilibrium prices at date $t$.

$$
\boldsymbol{q}_{t}=\frac{1}{d_{0, t+1}}\left(\boldsymbol{m}_{t}-\Psi_{t} \boldsymbol{S}_{t} \boldsymbol{\theta}\right)
$$


where

$$
\Psi_{t} \equiv \frac{1}{\int_{\ell}^{1} \frac{1}{\alpha^{h}+\phi_{t}^{h}} d h}
$$

$\Psi_{t}$ can be given an interpretation in terms of the average "effective risk aversion" across all traders at date $t$. It is proportional to the harmonic mean of the individual effective risk aversion coefficients $\alpha^{h}+\phi_{t}^{h}$.

The expression for equilibrium prices in (5) suggests that we can make some sense of the frequently heard statement during distressed episodes for the market that the "level of risk aversion has gone up." Our expression for equilibrium prices suggest that even if the basic risk aversion is constant (given by $\alpha^{h}$ ), the trader acts "as if" his risk aversion was fluctuating over time. It is in this sense that we can regard $\Psi_{t}$ as the average effective risk aversion of the traders.

The implementation of our model entails solving for the time series of $\left\{\Psi_{t}\right\}$. There are a number of subtleties in solving for $\Psi_{t}$, since the Lagrange multipliers $\lambda_{t}^{h}$ depend on the contemporaneous prices, and hence the solution of $\Psi_{t}$ involves solving a fixed point problem. The reader is referred to Daníelsson and Zigrand (2001) for the detailed solution method, and for the conditions for the existence of equilibrium. However, the solution for $\Psi_{t}$ can be given a relatively clean explicit characterization.

In order to state the solution for $\Psi_{t}$ explicitly, first consider the function $z e^{z}$. This is a non-monotonic function that has a minimum at $z=-1$. For $z<-1$, it is a decreasing function for $z$, while when $z>-1$, it is an increasing function of $z$. Consider the restriction of this function to the interval $(-\infty,-1]$. The inverse of this restricted function is defined for the interval $\left[-e^{-1}, 0\right)$, and takes values in $(-\infty,-1]$. This partial function is sometimes referred to as the non-principal branch of the Lambert correspondence. We denote this partial function as $F(\cdot)$. Then, the explicit solution for $\Psi_{t}$ can be stated as follows (see Daníelsson and Zigrand, 2001)

$$
\Psi_{t}= \begin{cases}\frac{1}{\ln \ell^{-1}} & \text { if } 0 \leq \kappa_{t} \leq \ell \ln \ell^{-1} \\ \frac{\kappa_{t}-\ell}{\kappa_{t} F\left(-\left(\kappa_{t}+\ell\right) e^{-1}\right)} & \text { if } \ell \ln \ell^{-1} \leq \kappa_{t} \leq 1-\ell\end{cases}
$$

where

$$
\kappa_{t} \equiv \sqrt{\frac{\boldsymbol{\theta}^{\prime} \boldsymbol{S}_{t} \boldsymbol{\theta}}{\bar{v}}}
$$

An equilibrium fails to exist when $\kappa_{t}>1-\ell$. The intuition for this lies in the interpretation of $\kappa_{t}$. The numerator is the total risk in the economy that must 
be borne by the traders as a whole, while the denominator is the maximum allowable risk. The total measure of traders is $1-\ell$, and equilibrium fails to exist when the per capita risk in the economy is too large for the traders to take on.

\section{Simulations}

We now come to the core of our paper, where we report the simulation results of our model. Having solved for the equilibrium prices, the latent data generation process (DGP) for the payoffs remains to be specified. The backward-looking updating rule used by the traders in our model means that the perceived DGP believed by the traders is different from the latent DGP. In particular, the fact that the distribution of the return process is latent and has to be forecast by a variance-covariance (VCV) method mirrors the actual market.

For our simulations, we chose a true payoff process that conformed to some of the stylized facts of financial returns. In order to take account of features such as volatility clustering, unconditional non-normality, and the relative size difference between returns and volatility, we specified a GARCH process, where the actual parameter values were chosen by calibration to fit some reasonable summary statistics. Thus, in the results we report below, the true payoffs are conditionally normal and unconditionally non-normal, and allow for volatility clustering. To ensure that initial parameter values do not affect the steady-state economy, we run the model for 500 simulations before recording any outcomes.

\subsection{Parameter Values}

The following parameter values for our model were used in the simulations, where $\hat{\boldsymbol{m}}$ is the mean of the latent payoff process, and $\hat{\boldsymbol{S}}$ is the unconditional covariance matrix of the latent payoff process. 


\begin{tabular}{ll}
\hline endowments & $\boldsymbol{\theta}=\left(\begin{array}{l}1.9 \\
0.5\end{array}\right)$ \\
\hline daily risk free rate & $r_{0}=1.00013$ \\
\hline decay factor & $\rho=0.97$ \\
\hline lowest risk aversion & $\ell=0.0011$ \\
\hline unconditional latent payoff distribution & $\hat{\mathbf{S}}=\left(\begin{array}{ll}0.6 & 0.25 \\
0.25 & 0.4\end{array}\right)$ \\
& $\hat{\boldsymbol{m}}=\left(\begin{array}{l}1.5 \\
1.2\end{array}\right)$ \\
\hline
\end{tabular}

The main results are presented in a series of figures. We first show the result of the typical run, and then we examine the impact of an exogenous shock to the true payoff process that is the equivalent of a large shock that may be expected once in ten years. Finally, we will report how we can track changes in the effective risk aversion, prices and volatility, perceived as well as actual. All figures are presented at the end of the paper.

\subsection{Analysis of a Run}

Given the sample economy, we set the risk constraint $\bar{v}=100$. The price evolution of the price of asset 1 is shown in Figure 1. The top panel tracks the price of asset 1 over time, where we have also superimposed the price path of the unregulated economy (where the VaR constraint does not bind). As we would expect for a positive beta asset, the price path of the regulated economy is below the price path of the unregulated economy. This is a natural consequence of the fact that the VaR constraint enters as an increment to the effective risk aversion of the traders. Increased effective risk aversion implies that prices are lower. The bottom panel shows the ratio of the unregulated price to regulated price. Note that the price differences can very substantial, ranging from zero to $40 \%$.

Figure 2 tracks elements of the traders' forecast covariance matrix of returns, $\Sigma_{t}$. The top panel reports the forecast variance for the return of asset 1 . Note how the imposition of the VaR constraint raises the forecast variance. The bottom panel shows this more explicitly by reporting the ratio of the unregulated variance to regulated variance for asset 1. Again, as with prices, the differences can be rather substantial. Figure 3 tracks the evolution over 
time of the average effective risk-aversion, $\Psi_{t}$. The unregulated $\Psi$ is 0.11 , but as can be seen, $\Psi_{t}$ fluctuated widely when the VaR constraint is imposed.

\subsection{A Shock}

We now examine the impact of a large exogenous one day shock to the payoff realisation of asset 1 . The magnitude of the shock equals the expected worst daily outcome in 10 years, i.e., the expected minimum of 2,500 realizations. This amount is -5.3 . In order to accurately gauge the impact, we repeated the exercise $10^{6}$ times and present the averages. The VaR constraint was set as $\bar{v}=1000$.

The effect of the shock is rather dramatic, as can be seen in Figure 4. There is a steep fall in the price after the shock, followed by a slow recovery. In particular, for the first few days after the shock the crisis deepens through the endogenous feedback in prices. Note, however, how the price path in the regulated economy follows a steeper decline and reaches a lower trough. The trough is deeper, and lasts longer in the regulated economy than in the unregulated one. The recovery of the price also takes longer. The bottom panel shows the ratio of prices across the two economies. The price ratio follows a rather interesting double-humped shape, in which the maximum difference in the prices follow rather late after the shock. At its maximum, the price ratio is almost $20 \%$.

Figure 5 plots the corresponding changes in the perceived volatilities in the economy in terms of the determinants of the forecast covariance matrices $\left\{\boldsymbol{S}_{t}\right\}$. The regulated economy with the VaR constraint has substantially higher values of $\left|\boldsymbol{S}_{t}\right|$, thereby inducing the traders to take more conservative trading decisions, and exacerbating the dynamics further. The bottom panel plots the ratio of the determinants across the two economies.

\subsection{Tracking Effective Risk Aversion}

One of the advantages of having an explicit model for the portfolio decisions of the traders is that it allows us to track the effect of a tighter VaR constraint through the economy. There are two ways of doing this. The first is through changes in the average effective risk aversion $\Psi_{t}$. Figure 6 plots the $\Psi_{t}$ realizations over time following the shock. The second way to show the effect of tighter VaR constraints is through the effects on prices and forecast variances. In figure 7 , we illustrate the impact of the severity of the VaR constraint on prices and perceived volatilities. 
The solid line is the ratio of the average prices $\left(q_{1}+q_{2}\right) / 2$ averaged across time. We can see that as $\bar{v}$ becomes smaller (so that the VaR constraint binds more tightly), the prices in the regulated economy fall further. We have already commented on the fact that when $\bar{v}$ becomes too small, equilibrium fails to exist in our model. For the parameters chosen in our simulations, this point is reached when $\bar{v}$ hits 12 . To the left of this point, equilibria do not exist. At the opposite extreme, we can see that as $\bar{v} \rightarrow \infty$, the constrained and unconstrained economies converge.

Also depicted in figure 7 is the ratio of determinants for the regulated and unregulated economies, as a function of $\bar{v}$. As we would expect, the differences in forecast volatility becomes very large when $\bar{v}$ is small. As $\bar{v} \rightarrow \infty$, the difference disappears.

In figure 8, we plot an investor's effective risk aversion $\alpha^{h}+\phi_{t}^{h}$. as a function of both the $\mathrm{VaR}$ constraint $\log \bar{v}$ but also of the population risk-aversion parameter $\alpha^{h}$ (recall that the population $\alpha^{h}$ is distributed uniformly on the interval $[\ell, 1])$. The kink in the surface marks the boundary where the VaR constraint "kicks in" (i.e. starts to bind). When a trader has a high value of $\alpha^{h}$, then the VaR constraint does not bind, and hence does not affect the economy.

\section{Conclusions}

The exercise in this paper has uncovered some unsettling side-effects of imposing value-at-risk constraints in an economy where traders follow backwardlooking belief revision rules. Far from stabilizing prices, the effect of such constraints is to induce behaviour that exacerbates the shocks further. 


\section{References}

Basak, S., And A. Shapiro (1995): "A General Equilibrium Model of Portfolio Insurance," Review of Financial Studies, 8, 1059-1090.

(2001): "Value-at-Risk Based Risk Management: Optimal Policies and Asset Prices," Review of Financial Studies, 14.

Basel Committee on Banking Supervision (1996): Overview of the amandment to the capital accord to incorporate market risk.

Brady, N. (1988): "Report of the Presidential Task Force on Market Mechanisms," Discussion paper, Government Printing Office, Washington D. C.

Crockett, A. (2000): "Marrying the micro- and macro-prudential dimensions of financial stability," Bank for International Settlements; http://www. bis.org/review/rr000921b.pdf.

DaníElsson, J. (2000): "The Emperor has no Clothes: Limits to Risk Modelling," Working paper, London School of Economics. www.RiskResearch.org.

DaníElsson, J., And J.-P. Zigrand (2001): "What Happens When You Regulate Risk? Evidence from a Simple Equilibrium Model," Working paper, London School of Economics. www.RiskResearch.org.

Gennotte, G., and H. Leland (1990): "Market Liquidity, Hedging, and Crashes," American Economic Review, 00, 999-1021.

Goodhart, C. A. E. (1974): "Public lecture at the Reserve bank of Australia," .

Grossman, S., And J.-L. Vila (1989): "Portfolio Insurance in Complete Markets: a Note," Journal of Business, 62, no. 4, 473-476.

Grossman, S., And Z. Zhou (1996): "Equilibrium Analysis of Portfolio Insurance," Journal of Finance, 51(4), 1379-1403.

LuCAs, R. E. (1976): "Econometric Policy Evaluation: A Critique," Journal of Monetary Economics, 1.2 Supplementary, 19-46.

Morris, S., And H. S. Shin (1999): "Risk Management with Interdependent Choice," Oxford Review of Economic Policy, Autumn 1999, 52 - 62, reprinted in the Bank of England Financial Stability Review, 7, 141 - 150, http://www . bankofengland.co.uk/fsr/fsr07art5.pdf. 
Persaud, A. (2000): "Sending the herd off the cliff edge: the disturbing interaction between herding and market-sensitive risk management practices," Larosière prize winning essay, Institute for International Finance, http://www . erisk. com/news/features/persaud.pdf.

RiskMetrics Group (1999): "RiskMetrics Technical Manual," http://www.riskmetrics.com/research/techdocs/index.cgi.

Shiller, R. (1987): "Investor Behavior in the October 1987 Stock Market Crash: Survey Evidence," Discussion paper, NBER discussion paper 2446.

The Economist (2000): "Is the end in Sight?," The Economist. 

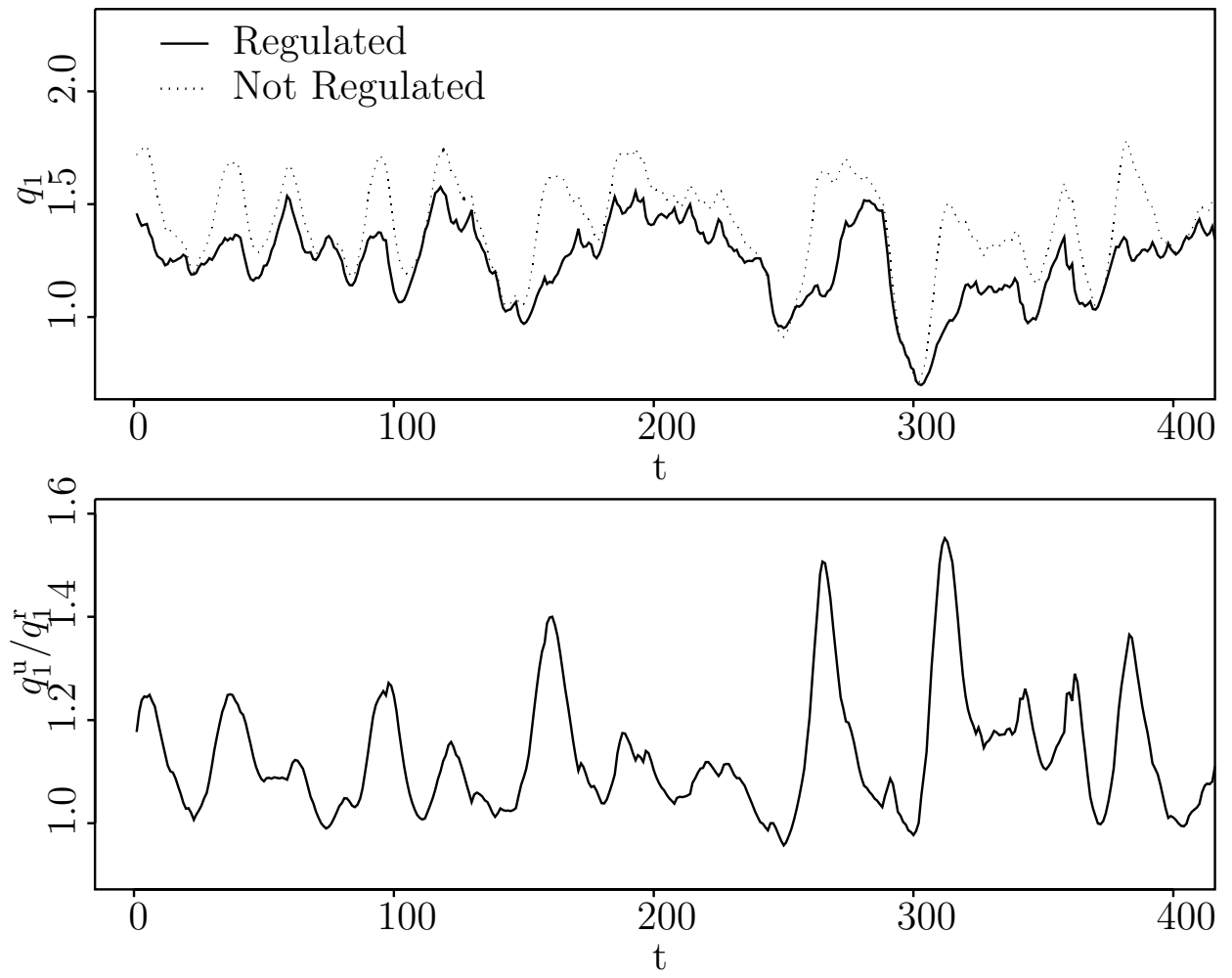

Figure 1: Impact on Prices

400 realizations of the prices of asset one in the sample economy with risk constraint $\bar{v}=100$. The lower figure shows the ratio of the regulated to unregulated prices. Note it is always greater than one, indicating that the regulated economy has lower prices. 

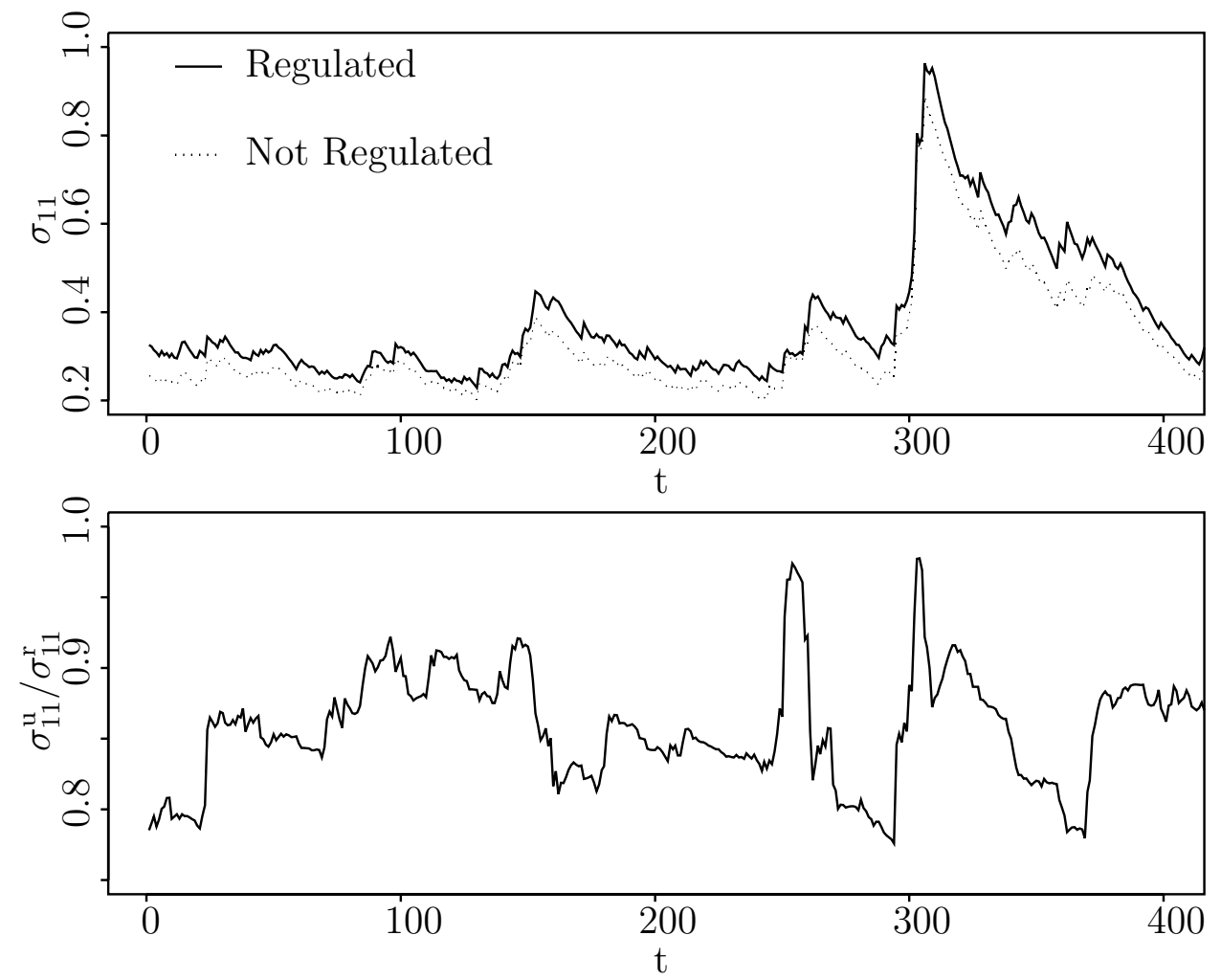

Figure 2: Impact on Perceived Return Variance

400 realizations of the volatility of asset one in the sample economy with risk constraint $\bar{v}=100$. The lower figure shows the ratio of the regulated to unregulated volatilities. Note it is always less than one, indicating that the regulated economy is more volatile. 


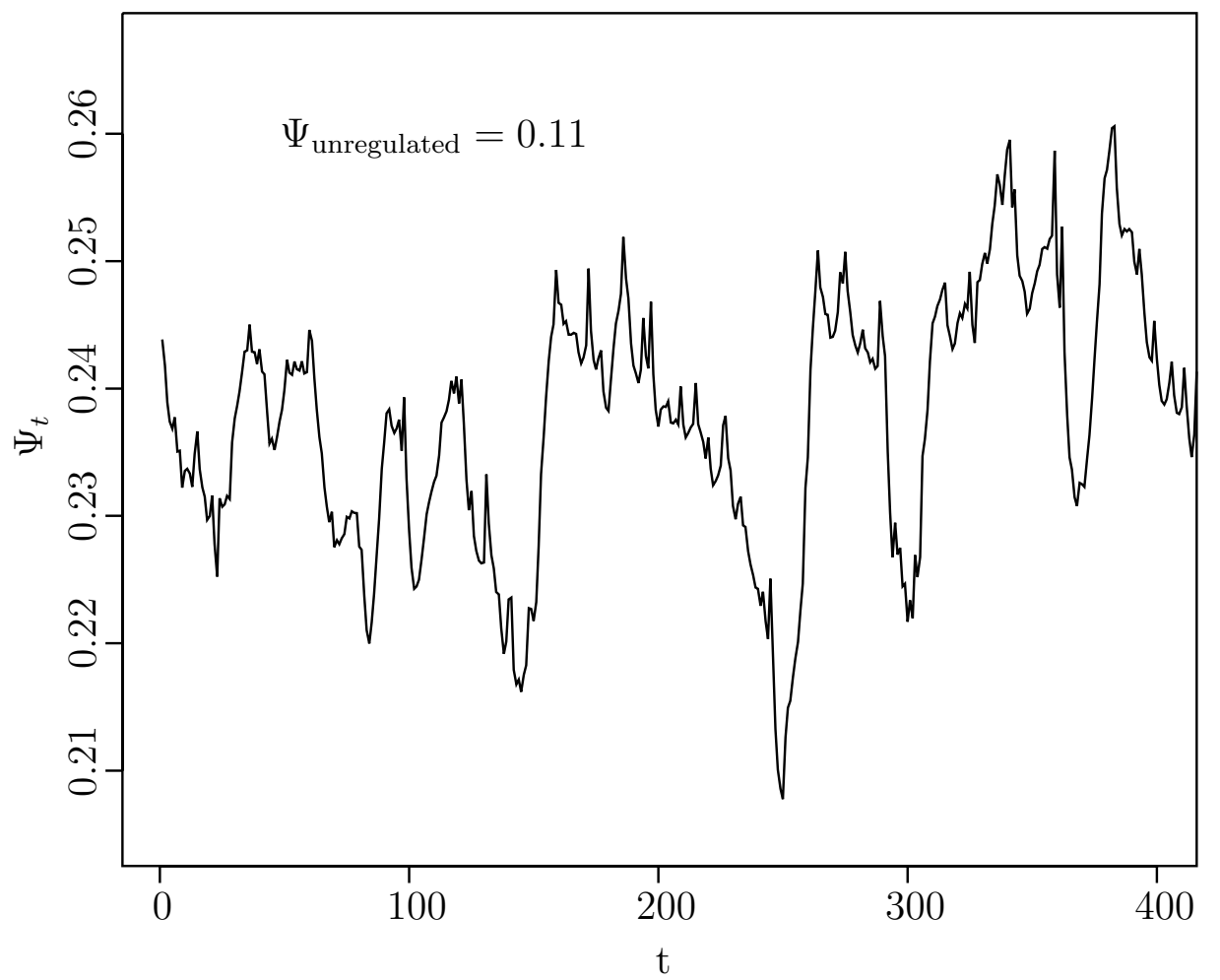

Figure 3: $\Psi_{t}$

400 realizations of $\Psi_{t}$ in the constrained sample economy with risk constraint $\bar{v}=100$.

The unregulated economy has $\Psi=0.11$. 

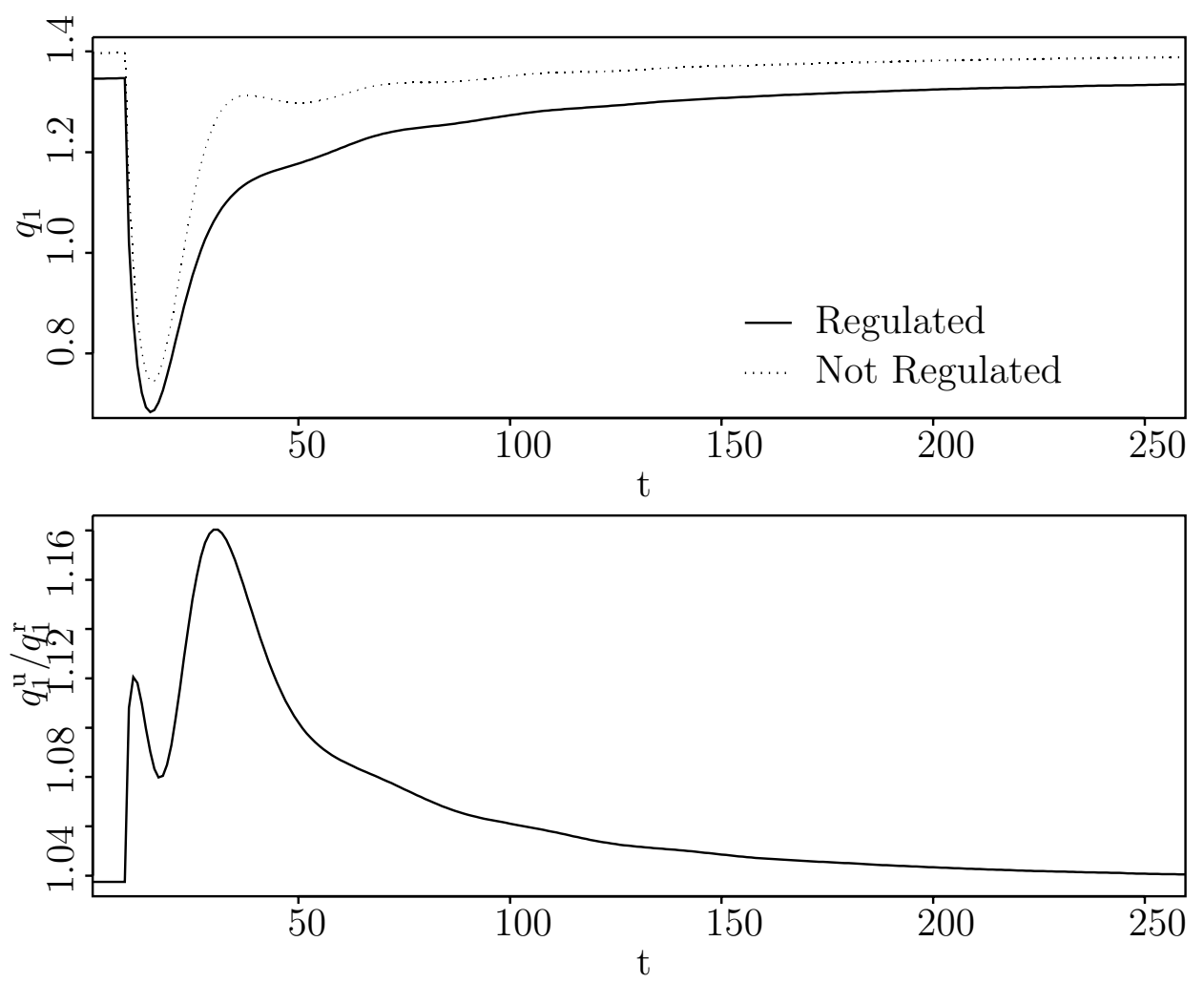

Figure 4: 10 Year Shock to Payoffs. Price Impact

The result of a single shock equaling the expected largest 10 year (2500 obs.) adverse shock to payoffs of asset one. Economy starts in steady state, and the exercise is repeated $10^{6}$ times. The lower figure shows the ratio of the regulated to unregulated prices. Note it is always larger than one, indicating that the regulated economy has lower prices. Further, the shock impact is larger, and the adjustment to steady state takes longer in the regulated economy. 

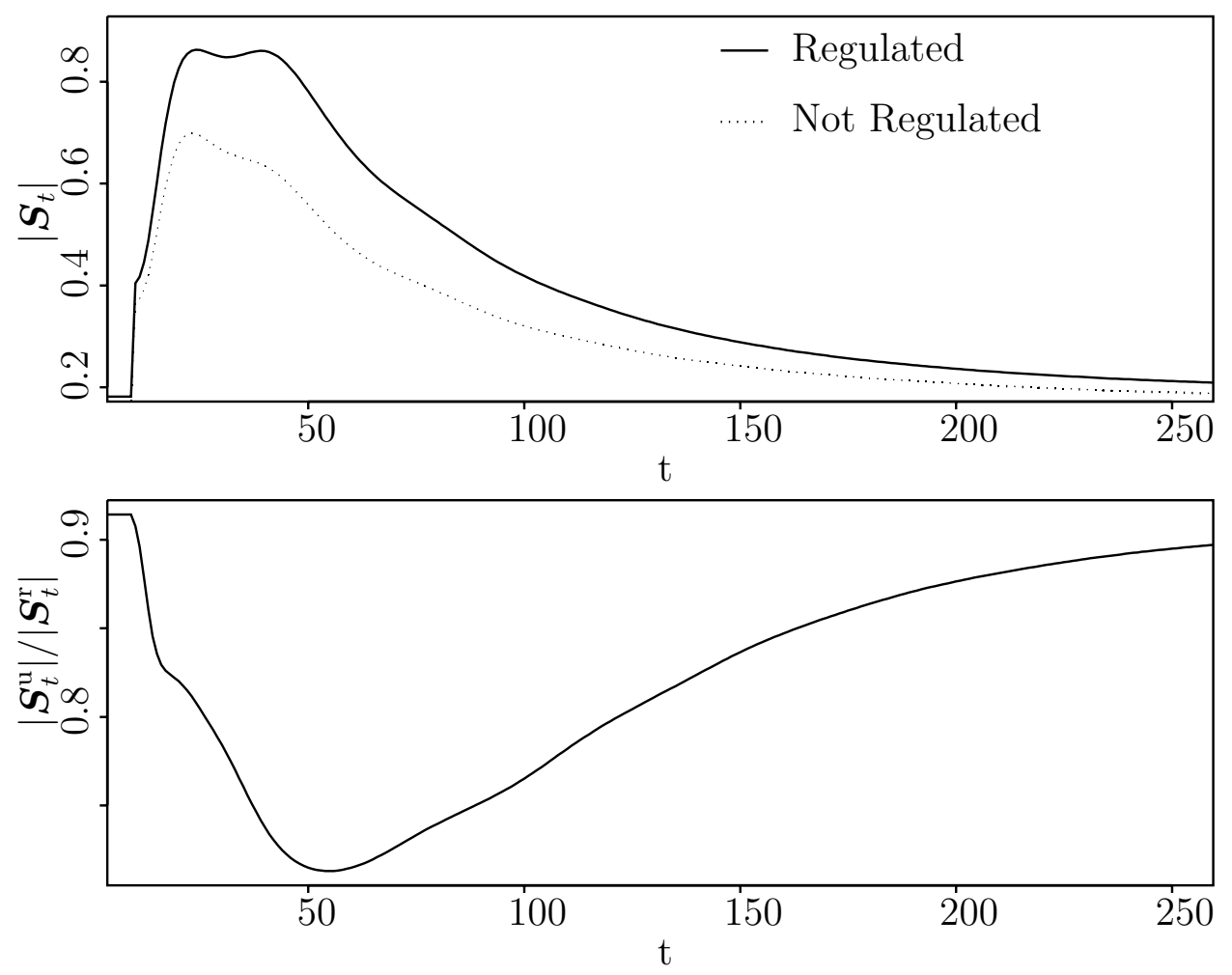

Figure 5: 10 Year Shock to Payoffs: Perceived Vol. Impact

The result of a single shock equaling the expected largest 10 year (2500 obs.) adverse shock on payoffs on the forecasted covariance matrix. Economy starts in steady state, and the exercise is repeated $10^{6}$ times. The lower panel shows the ratio of the unregulated covariance matrix to the regulated covariance matrix. Note it is always less than one, indicating that the regulated economy is more volatile. Further, the shock impact is larger, and the adjustment to steady state takes longer in the regulated economy. 


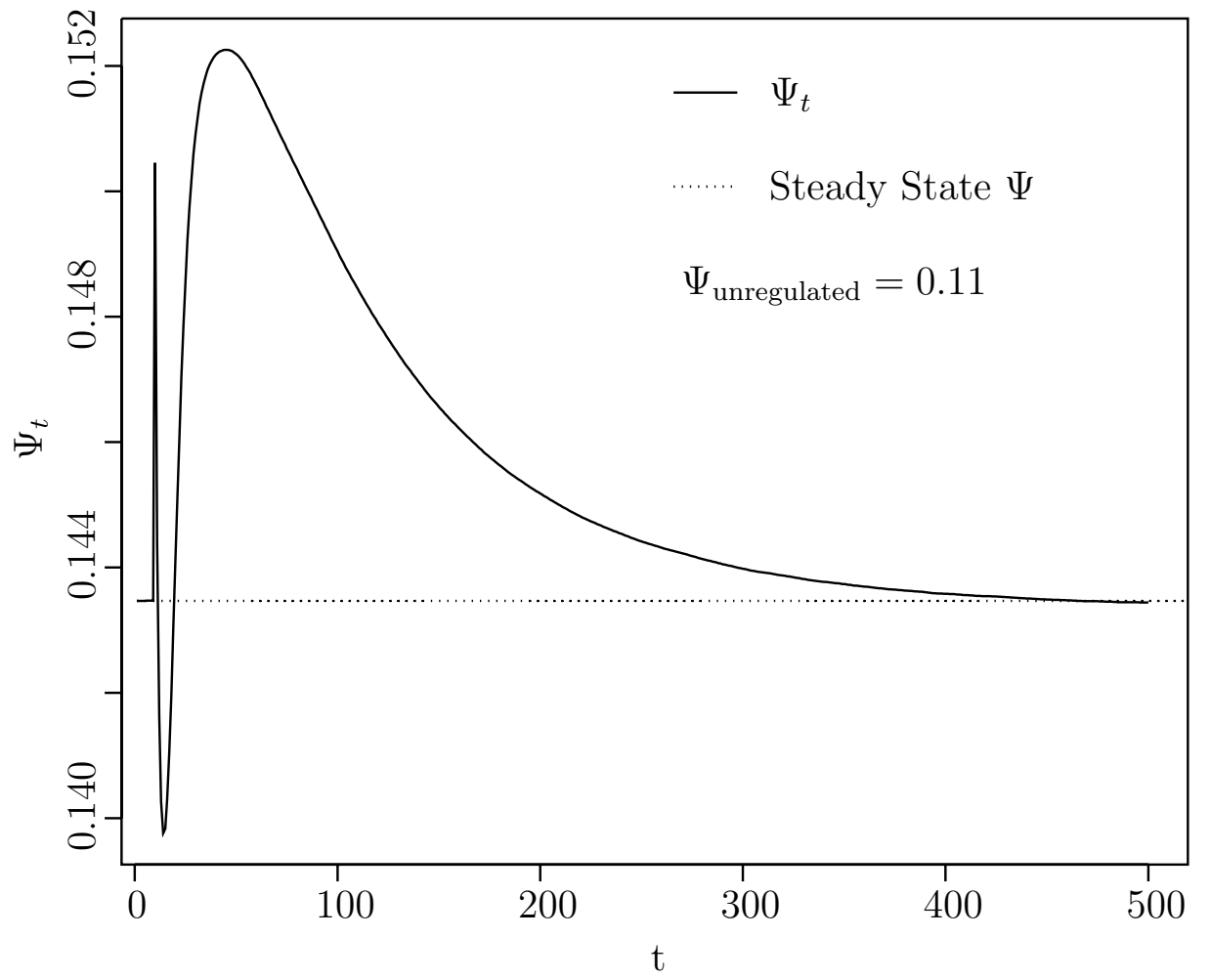

Figure 6: Max 10 Year Shock to Payoffs: Impact on $\Psi$

The result of a single shock equaling the expected largest 10 year (2500 obs.) adverse shock to payoffs on $\Psi$. Economy starts in steady state, and the exercise is repeated $10^{6}$ times. 


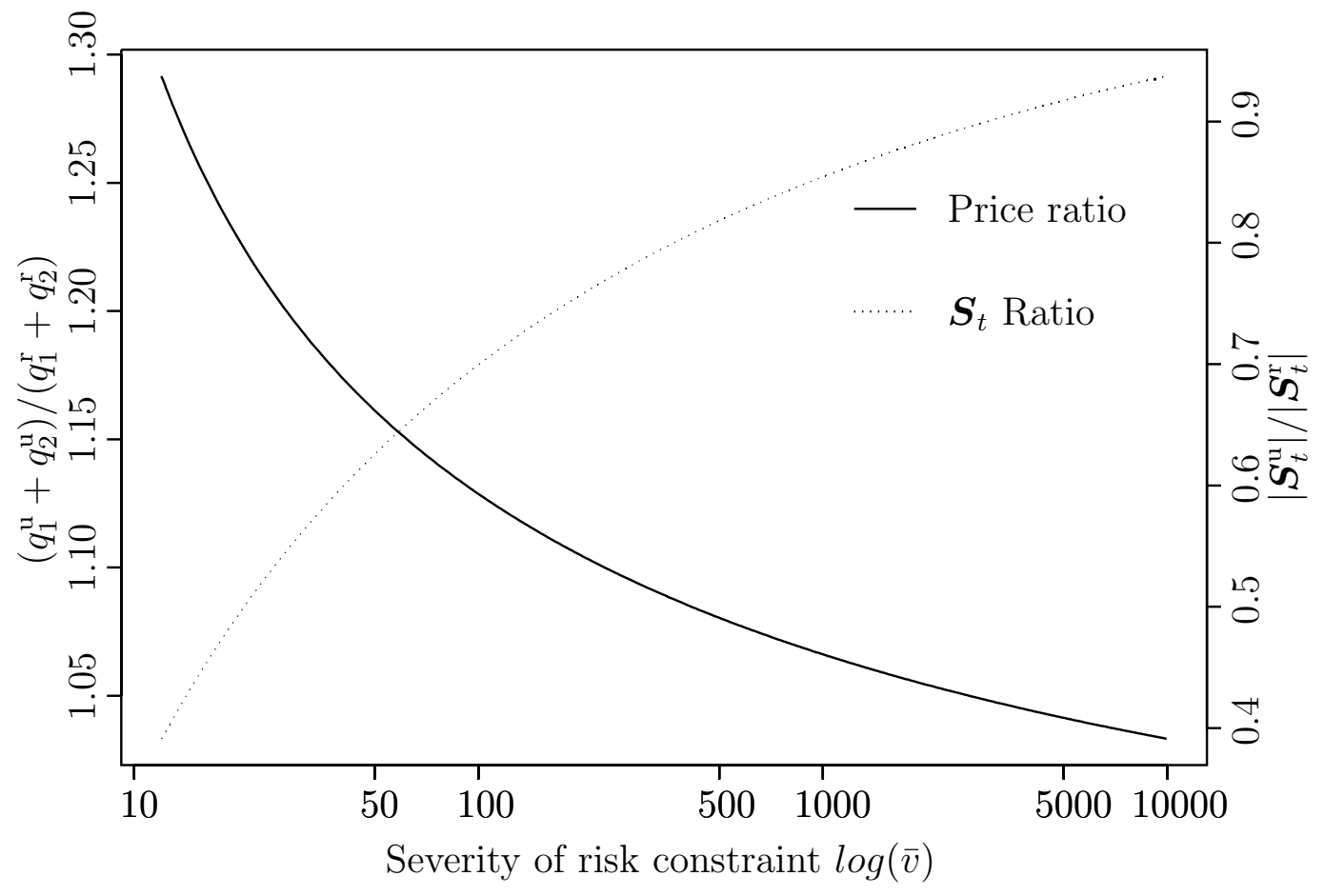

Figure 7: Increasingly Restrictive Risk Constraint

Impact on the sample economy of adjusting the risk constraint, $\log (\bar{v})$. At values $\log (\bar{v})<$ 11 equilibrium is no longer defined, and at $\log (\bar{v})=\infty$ the constraint is no longer binding. The figure shows the ratio of the unregulated average prices to the unregulated prices, and the same ratio for the perceived covariance matrix. Note how as $\log (\bar{v}) \rightarrow=\infty$, the ratios tend to one. 


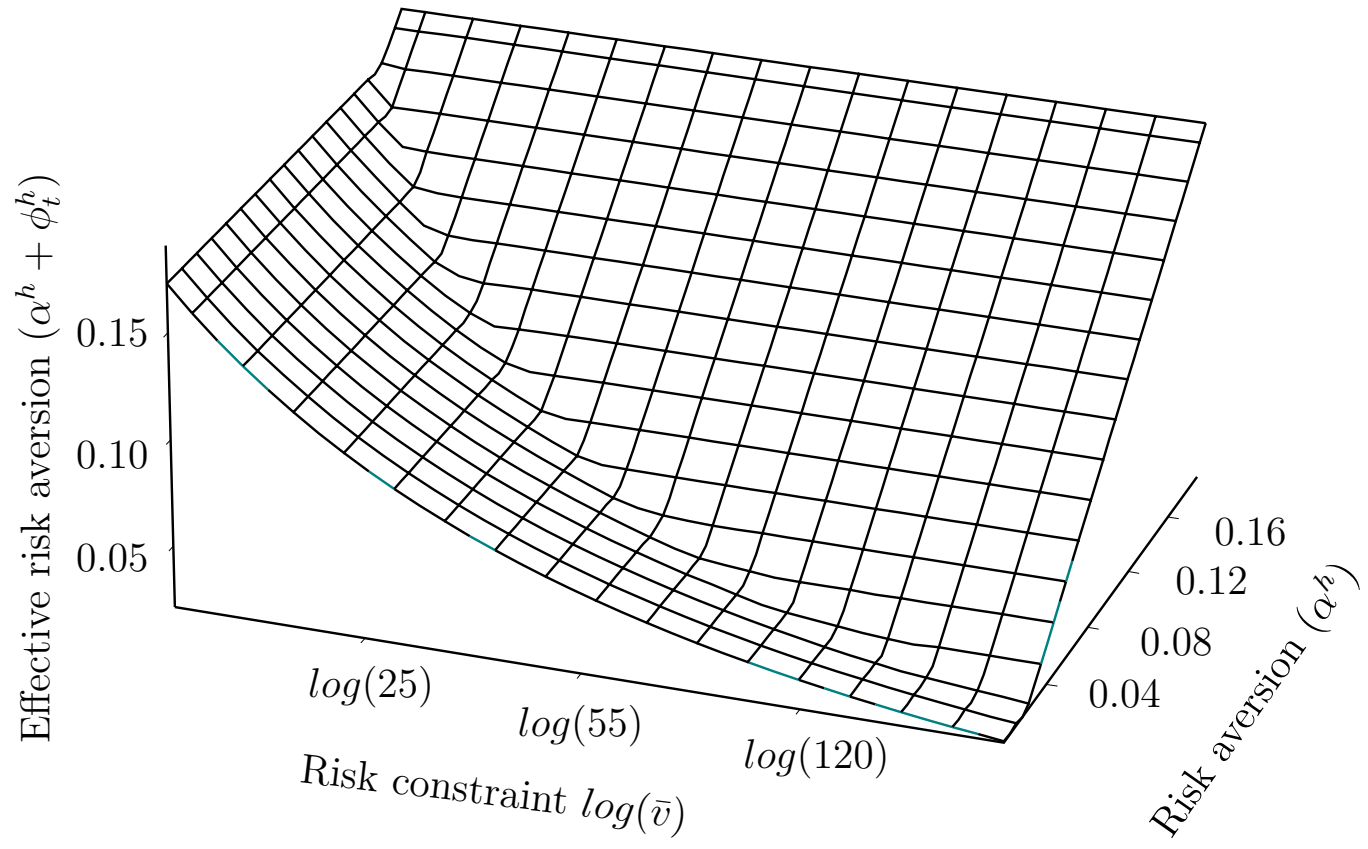

Figure 8: Effective Risk Aversion

Impact on effective risk aversion, $\left(\alpha^{h}+\phi_{t}^{h}\right)$, of adjusting risk constraint, $\log (\bar{v})$, for traders with varying risk aversion, $\left(\alpha^{h}\right)$. 